Review

\title{
Preventive Measures and Management of Catheter- Associated Urinary Tract Infection in Adult Intensive Care Units in Saudi Arabia
}

\author{
Najla A. Obaid \\ College of Pharmacy, Umm Al-Qura University, Makkah, Saudi Arabia
}

\section{ARTICLE INFO \\ Article History \\ Received 12 October 2020 \\ Accepted 15 April 2021 \\ Keywords \\ Catheter-associated urinary tract infection \\ device associated infection \\ preventive measures \\ good clinical practice}

\begin{abstract}
Catheter-associated Urinary Tract Infection (CAUTI) has been studied worldwide as part of device-related healthcare infection. CAUTI is one of the most commonly reported infections in the literature, which occurs during clinical practice in Intensive Care Units (ICUs) and non-ICU departments. Many studies have covered the CAUTI rate in Saudi Arabia mainly in the ICU covering single or multiple hospitals as surveillance for device-related infections in the ICU. Few studies have conducted an interventional approach that examined the implementation of infection control protocols and then compared them to international practice as a standard. This review aims to explore the literature to provide insight into the infection control practices that have been reported in Saudi Arabia during the last two decades on the preventive measures and clinical consequences of CAUTIs. Very few studies have recorded the pattern of resistant microorganisms that burdens clinical practice in ICUs concerning CAUTIs. Only one study compared the type of catheter materials and discussed the effect of these materials on reducing CAUTIs. It is essential to cover catheter utilization and to understand how a sufficient infection control protocol with educational programs for healthcare personnel can transform practice, for the better, regarding CAUTI rates in Saudi hospitals. There is a demand for more interventional and epidemiological studies on the causes and factors affecting the rate of CAUTI in the area. Studies may help reduce the CAUTI incidence rate, which consequently reduces the costs and morbidity associated with this type of infection and other healthcare-related infections.
\end{abstract}

(C) 2021 The Author. Published by Atlantis Press International B.V.

This is an open access article distributed under the CC BY-NC 4.0 license (http://creativecommons.org/licenses/by-nc/4.0/).

\section{INTRODUCTION}

Hospital-acquired Infections (HAIs) are considered complications of medical therapy and are due to many factors. The unnecessarily prolonged length of hospital stay, use of invasive devices, and improper use of antimicrobial therapy are believed to be factors that contribute to a higher rate of HAIs [1]. According to Centers for Disease Control and Prevention (CDC) estimates, approximately one in 31 hospitalized patients experienced a HAI in the United States and $3.2 \%$ of patients (394 of 12,299 patients) in a survey conducted in 2015 [2]. The most prevalent HAIs related to invasive devices are central line-associated bloodstream infections, Catheterassociated Urinary Tract Infections (CAUTIs), and ventilatorassociated pneumonia. Most of these infections are caused by multidrug resistant organisms [3]. CAUTIs are considered the most predominant HAIs, and according to Magill et al. [2], 18.7\% of surveyed patients reported a CAUTI. Approximately, $80 \%$ of all UTIs during hospitalization are caused by an indwelling urethral catheter [4]. Clinical consequences such as prostatitis, pyelonephritis and cystitis in patients with CAUTI are the major concern within the ICU domain. A prolonged hospital stay attributable to device-related infections is an avoidable situation, but whenever it occurs, there is an increase in healthcare costs and mortality for patients [5].

"Email:naobaid@uqu.edu.sa
Clinicians and nursing staff in collaboration with the infection control departments play a pivotal role in reducing and/or preventing these types of infections in hospitalized patients in ICU and non-ICU departments. Several strategies and protocols have been developed by infection control departments to prevent CAUTI infections. For example, improvement in compliance with hand hygiene demonstrated to be effective in reducing HAIs in the ICU as part of implementing infection surveillance with prevention programs [6].

This review explores observational and surveillance studies conducted to report CAUTIs, particularly in intensive care units in health institutes, and preventive measurements, clinical consequences and factors affecting the rate of CAUTI in Saudi Arabia during recent decades.

\section{MATERIALS AND METHODS}

This review was performed by searching several electronic databases (Medline via PubMed, Google Scholar, and Scopus) using keywords [CAUTI, Device-associated Infection (DAI) and Saudi Arabia]. Starting with the search with "CAUTI", which showed the results of 838 studies in PubMed, 36800 results in Google Scholar and 763 documents in Scopus. After adding "catheter-associated 
urinary tract infection" AND "Saudi Arabia" to the search tool, PubMed showed 13 documents, Google Scholar 11 documents and Scopus 10 documents with the exclusion of some studies that appeared in the search results with health-acquired infection and non-CAUTI. The process of selection articles for this review is illustrated in Figure 1.

\section{INFECTION CONTROL STRATEGY AND THE EFFECT OF THE IMPLEMENTATION OF PROTOCOLS FOR THE MANAGEMENT OF CAUTI IN SAUDI ARABIA}

Suitable handling, insertion and removal of the urinary catheter have a major effect on preventing and controlling CAUTIs. On the one hand, factors such as the duration of catheterization, aseptic techniques during insertion of the catheter, and handling of urinary bags have a significant impact on the incidence of CAUTI $[7,8]$. On the other hand, the overreporting of CAUTI events may be due to inappropriate collection and testing of urine specimens, which consequently leads to the treatment of false-positive cultures, increasing the overuse of antimicrobial agents [9]. The implementation of protocols for DAI has a significant impact on reducing these types of infections, including CAUTI [10].

Hand Hygiene $(\mathrm{HH})$ is the first effective strategy followed by infection control to minimize HAIs and to ensure patient safety in several studies worldwide [11-13]. In Saudi Arabia, the implementation of a multimodal strategy with improving $\mathrm{HH}$ in a university-affiliated
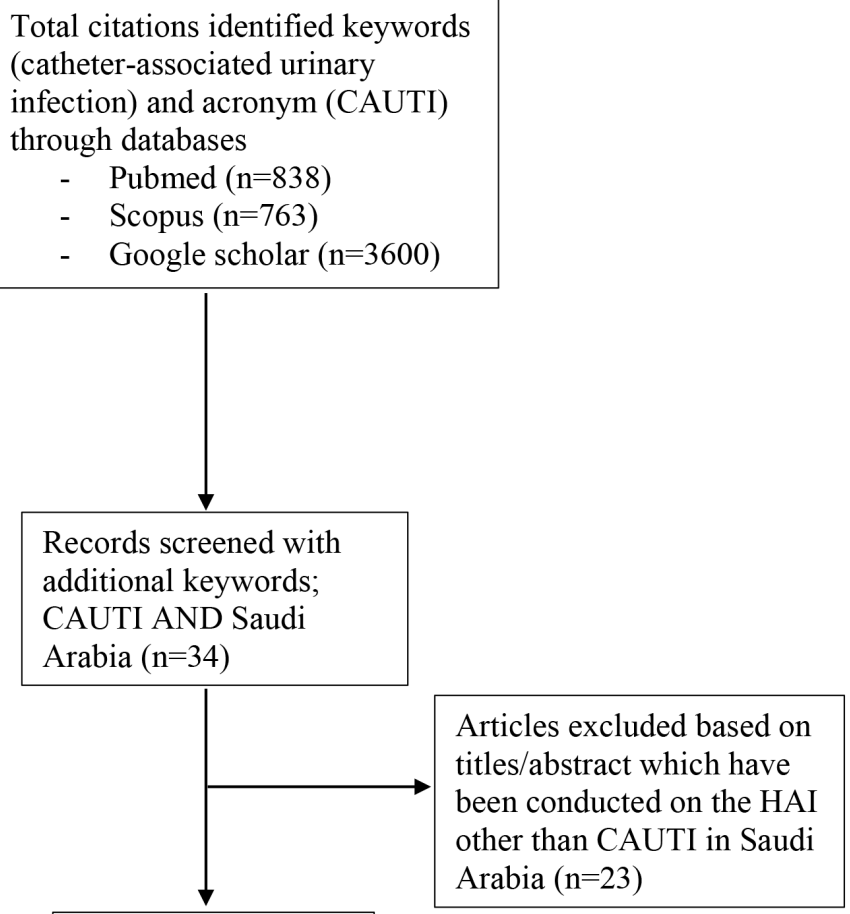

Articles included in this review $(\mathrm{n}=11)$

Figure 1 Summary of the research method used in this review to explore CAUTI preventive measures and incidence rate in Saudi Arabia. hospital at AL Khobar, Saudi Arabia, reduced the rate of CAUTI from 3.73 in 2014 to 2.21 in 2015 , which then further decreased to 1.75 in 2016 [14]. The study suggested that educational intervention and enhancement of $\mathrm{HH}$ compliance as part of a multicomponent strategy implemented in healthcare staff practice reduce HAIs.

One study in Saudi Arabia concluded that the most beneficial technique of infection control practice to reduce CAUTI is removing the catheter as soon as possible based on the recommendation of Society for Healthcare Epidemiology of America/Infectious Diseases Society of America practice [15]. The study was conducted between 2011 and 2013 at King Abdulaziz Specialist Hospital in Taif, Saudi Arabia. The expedited removal of urethral catheters as soon as possible reduced the rate of CAUTI from 3.5 to 2.9 per 1000 catheter/days in 2011 and 2012, respectively [15]. Further reduction in CAUTI was achieved and the rate reached 2.2 per 1000 catheters/day in 2013 [15]. The reductions in CAUTI incidence were due to applying the technique of removing unnecessary urinary catheters. Another study in Saudi Arabia conducted at the Saudi Aramco Medical Services Organization in the eastern province of Saudi Arabia showed that a higher DAI rate was catheterassociated urinary infection at the ICU wards [10]. This study indicated that CAUTI was the most common DAI, with a percentage of $42.2 \%$ during the period from 2004 to 2011 [10]. After implementing the CAUTI protocol to control HAIs, the CAUTI rate was reduced from 6.75 to $3.41(p=0.04)$, which indicated a linear trend in the reduction rate of CAUTI from the beginning to the end of the study period. This was an 8-year prospective study based on one healthcare center but included multiple ICUs.

Another study on the impact of applying interventions to control CAUTIs was conducted by collecting data from adult ICUs over 9 years in a tertiary hospital with 28 beds in the western region of Saudi Arabia. CAUTIs were reported at a high rate (2.3 per 1000 device/day) between 2008 and 2010, which initiated the implementation of an infection control protocol for CAUTI in the ICU for that hospital [16]. After applying the protocol in 2011, the CAUTI rate was reduced to 0.3 per 1000 device/day and then was further reduced to 0.1 and 0.2 in 2015 and 2016, respectively [16]. This reduction was mainly based on preventive interventions and strict compliance with the strategy implemented.

Most of the studies showed that the rate of CAUTI as part of DAI in ICUs is high, and Saudi Arabia still faces significant challenges for infection control practice [17]. Benchmarking the infection control practice with a well-established strategy such as CDC/National Healthcare and Safety Network (NHSN) validates the surveillance of the HAI rate [17]. A worldwide online platform to establish surveillance by International Nosocomial Infection Control Consortium (INICC) reported data related to the DAI-HAI rates and published a summary from January 2012 to December 2017 [18]. This report collected data for DAI, including CAUTI, for hospitals from 42 different countries and involved patients admitted to the ICU, and Saudi Arabia was part of this data collection. Detailed data from the patient's specific device collection, microbiological profile and clinical profiles were collected using the INICC Surveillance Online System [18]. CAUTI was one of the types of DAI data collected for rates and microbiological profiles, including bacterial resistance and mortality. The number of patients, CAUTI cases, urinary catheter days, types of pathogens and resistance percentage were collected from 11 different ICU wards, 
including Saudi Arabia. From all these ICU wards, the number of ICUs reported for CAUTI was 455 [18]. This report has provided several insights into DAI and device utilization and the rates and ratios for these infections worldwide; however, it does not offer information on the intervention and implementation approaches in each country and their hospitals.

Detailed protocol and assessment tools for preventing CAUTI were examined in a quasi-experimental study performed in a surgical ICU at King Fahad Hospital in Madinah [19]. This interventional study was based on assessing the performance of 30 nurses on two groups of patients $(n=80)$ with urinary catheters (a control group and an intervention group). The development of symptom reports to assess CAUTI, observational checklists and care protocols were tools implemented for the intervention group of the patients [19]. These tools were developed by the investigators based on their review of the relevant literature. The authors reported a reduction in the CAUTI percentage (15\%) for the intervention group compared to the control group (85\%) [19]. This study also stated that $\mathrm{HH}$ is one of the most important items that nurses should adopt in the prevention of CAUTI during the handling and insertion of the urinary catheter. Educational programs for the nurses and effective training on the good practices involved in the infection control protocol have been improved for the nurses enrolled in this interventional study. A significant reduction in the CAUTI rate after the implementation of evidence-based recommendations was established after an interventional study of non-ICU wards in a large tertiary hospital in Riyadh [20]. The study by Ghazal et al. [20] implemented a protocol, called the "bladder bundle", in a general ward (non-ICU) and used a model of NHS hospitals in England. The study showed a successful rate in reducing the infection in ICU wards. The study also conducted a prospective interventional project to decrease CAUTI in two non-ICU units [20]. Compliance with a detailed protocol is the key component of the prevention program that hospitals should adopt to reduce and to eliminate the development of infections related to urinary catheterization.

\section{REPORTING THE INCIDENCE RATE AND FACTORS INFLUENCING CAUTI IN SAUDI ARABIA}

The CAUTI rate has been reported in many studies worldwide [21]. One large study has reported the CAUTI rate of DIA through INICC by pooling data from 50 countries from 2010 to 2015 [18]. There are limited reports in the literature investigating and presenting the prevalence of CAUTIs in the long term and the factors influencing infection with epidemiological data for CAUTIs in Saudi Arabia. In Table 1, the studies performed in Saudi Arabia and the reported incidence rate (rate of infection per 1000 catheter days) are illustrated with the study settings. As part of the official surveillance report in each hospital in Saudi Arabia, there is a demand to investigate CAUTI in various ICU wards in hospitals. To reduce the infection rate and costs related to this infection, systematic collection of data, and improvement in techniques and infection control practices, collectively, would be a major approach to improve the quality of the health care system. Within three hospitals from three different Arabian Gulf countries, the CAUTI rate (3.2 per 1000 catheters/day) was investigated over 6 years, and compliance with the implemented protocols was $65 \%$ overall [22]. This is the only study that investigated the CAUTI rate within multiple healthcare centers in different Gulf countries. A study explored the DAI in a selected group of Ministry of Health hospitals $(n=12)$ in Saudi Arabia over the period 2013-2016 [17]. The study stated that CAUTI was the second most common DAI (28.4\%) in the ICU of these hospitals and occurred at 2.3-4.4 per 1000 devices/day [17]. This rate is high compared to the ratios from NHSN, which urges

Table 1 The incidence rate and factors of CAUTI reported in Saudi Arabia

\begin{tabular}{|c|c|c|c|}
\hline Type of study & CAUTI rate & Location & References \\
\hline Observational and surveillance & 3.2 per 1000 catheter day & $\begin{array}{l}\text { Three GCC countries (Saudi Arabia, } \\
\text { Oman and Bahrain) }\end{array}$ & [22] \\
\hline Observational and surveillance & 2.3-4.4 per 1000 device/day & $\begin{array}{l}12 \text { hospitals of Ministry of Health } \\
\text { in Saudi Arabia }\end{array}$ & [17] \\
\hline $\begin{array}{l}\text { Prevalence of antibiotic resistance for } \\
\text { CAUTI and non CAUTI }\end{array}$ & $60 \%$ beta-lactamases producer in CAUTI & Al-Dawadmi, Saudi Arabia & [27] \\
\hline $\begin{array}{l}\text { Observational, single-blinded and } \\
\text { prospective study }\end{array}$ & $\begin{array}{l}90 \% \text { reduction of CAUTI in the noble } \\
\text { metal alloy catheter group }\end{array}$ & $\begin{array}{l}\text { University affiliated hospital at } \\
\text { AL Khobar, Saudi Arabia }\end{array}$ & [28] \\
\hline Interventional study & $\begin{array}{l}2014-2016 \text { (3.73-2.21 then further } \\
\text { decrease in to } 1.75 \text { respectively) per } \\
\text { 1000-catheter days }\end{array}$ & $\begin{array}{l}\text { University affiliated hospital at } \\
\text { AL Khobar, Saudi Arabia }\end{array}$ & [14] \\
\hline Prospective and surveillance study & CAUTI 8.18 per 1000 device-days & $\begin{array}{l}\text { Saudi Aramco Medical Services } \\
\text { Organization }\end{array}$ & [10] \\
\hline Prospective study & $\begin{array}{l}3.5,2.9 \text { and } 2.2 \text { per } 1000 \text { catheter-days } \\
\text { in } 2011-2013 \text { respectively }\end{array}$ & $\begin{array}{l}\text { King Abdul Aziz Specialist Hospital, } \\
\text { Taif, Saudi Arabia }\end{array}$ & [15] \\
\hline $\begin{array}{l}\text { Surveillance study of pooled data reported from } \\
45 \text { countries including Saudi Arabia }\end{array}$ & 5.1 vs 1.7 per 1000 catheter-days & Ministry of Health & {$[18]$} \\
\hline Quasi-experimental, interventional study & N/A & $\begin{array}{l}\text { King Fahad Hospital in Madinah, } \\
\text { Saudi Arabia }\end{array}$ & [19] \\
\hline $\begin{array}{l}\text { Prospective interventional study in non-ICU } \\
\text { settings }\end{array}$ & $\begin{array}{l}5.03 \text { per } 1000 \text { catheter-days in } 2008 \text { and } \\
1.92 \text { per } 1000 \text { catheter-days in } 2009\end{array}$ & $\begin{array}{l}\text { King Fahad Medical City in Riyadh, } \\
\text { Saudi Arabia }\end{array}$ & [20] \\
\hline $\begin{array}{l}\text { Surveillance study of pooled data of device associated } \\
\text { nosocomial infection reported from five hospitals } \\
\text { by using online surveillance system prospectively }\end{array}$ & 4.7 per 1000 urinary catheter day & $\begin{array}{l}\text { Seven ICU of five hospitals in five } \\
\text { different cities in Saudi Arabia }\end{array}$ & [29] \\
\hline
\end{tabular}

GCC, Gulf Cooperation Council. 
effective monitoring and management of infection control practices regarding DAI and CAUTI. Even though the authors in this cited study claimed that Saudi Arabia has limited studies regarding DAI overall and the incidence rate in general hospitals, such data will be vital for the benefit of the healthcare system to improve patient care. The goal is to identify health care settings with a high incidence rate of infection related to device insertion, which is considered a source of infection in hospitals that can be controlled by following good practices.

One recent study identified the microorganisms that contributed to the DAI as a source of transmitting HAIs. This study explored the microorganisms that contributed to HAIs in the ICU as an observational study in a relatively small general hospital in the central area of Saudi Arabia [23]. The study also audited the infection control protocol at the site of the study for device-associated practice in the ICU and recommended further effective strategies to control the infection and to reduce antibiotic resistance cases [23]. CAUTI, specifically in the ICU, was not covered enough in the literature to provide recommendations for the treatment protocols that help to manage the infection and to prevent the development of resistant pathogens.

One study stated that Escherichia coli (37.8\%), Klebsiella pneumonia (6.4\%) Pseudomonas aeruginosa (11.7\%), Candida spp. (14.4\%), Proteus mirabilis (4.8\%), Enterobacter spp. (2.1\%) and Acinetobacter calcoaciticus (3.2\%) were detected isolates caused UTIs as healthcare-associated infections without any specification to the CAUTI [24]. Over the last decade, there have been insufficient reports of bacterial strains that participate in the failure of antibiotic treatment and the development of antibiotic resistance for CAUTI cases. It is critical to report the resistance pattern of DAIs as part of HAIs, which have increased and become a real issue with multidrug resistance and pandrug resistance. Inadequate investigation and inappropriate management of sampling methods for the diagnosis of CAUTI could lead to burdens on both patients and health institutions [25]. The inappropriate reporting and collection of urine samples from catheters could cause overreporting of CAUTI incidence and increase the cost of managing the infection in the health institute. There are inadequate records for the microorganism strains and resistance patterns related to CAUTI in Saudi Arabia.

Effective treatment is directly linked to the appropriate investigation of the source of infection and the presence of specific symptoms. Overprescribing antibiotics in the absence of symptoms with bacteria should be avoided, and this practice commonly resulted from incorrect sample collection and/or lack of proper implementation of infection control programs for CAUTI [26]. Beta-lactamaseproducing Enterobacteriaceae are the major type of pathogens that were isolated from CAUTI in a single health center in Saudi Arabia [27]. The study found that extended-spectral beta-lactamase and other resistance features were higher in catheterized patients from different wards in the hospital [27]. To the best of our knowledge, this is the only study performed in the area to investigate the isolates that are related to CAUTI from the DAI of HAI.

The type of catheter may also play an important role in reducing the incidence of CAUTI for patients in the ICU $[8,28]$. A randomized single-blinded prospective study evaluated the efficacy of noble metal alloy-coated catheters in 30 ICU patients compared to 30 patients with the standard catheter (no coating) in King Fahad
Hospital in Saudi Arabia [28]. The coated noble metal alloy catheter significantly reduced CAUTI for the patients after 3 days of use [28], which is the only observational study that compared two different catheter types in clinical settings in the area. Further investigations on the effect of the catheter type by preclinical and clinical studies are needed.

\section{CONCLUSION}

This review concludes that urinary catheter utilization programs and the implementation of detailed protocols to prevent CAUTI are essential and insufficiently covered in Saudi Arabia. Systematic investigations throughout multiple hospitals to examine the current practices in Saudi Arabia are an important tool to control DAICAUTIs. Not enough epidemiological and observational studies in the area that investigated the causative pathogens and resistance patterns related to CAUTI in Saudi Arabia have been pointed out here. It will be vital to produce additional interventional studies assessing the implementation of tools and educational programs to train the health care staff that handle these devices in hospitals.

\section{CONFLICTS OF INTEREST}

The author declares no conflicts of interest.

\section{ACKNOWLEDGMENT}

The author would like to thank the Deanship of Scientific Research at Umm Al-Qura University for supporting this work by Grant Code: 19-MED-1-03-006.

\section{REFERENCES}

[1] Al-Tawfiq JA, Tambyah PA. Healthcare associated infections (HAI) perspectives. J Infect Public Health 2014;7;339-44.

[2] Magill SS, O'Leary E, Janelle SJ, Thompson DL, Dumyati G, Nadle J, et al. Changes in prevalence of health care-associated infections in U.S. hospitals. N Engl J Med 2018;379;1732-44.

[3] Khan HA, Baig FK, Mehboob R. Nosocomial infections: epidemiology, prevention, control and surveillance. Asian Pac J Trop Biomed 2017;7;478-82.

[4] Niël-Weise BS, van den Broek PJ. Urinary catheter policies for short-term bladder drainage in adults. Cochrane Database Syst Rev 2005;CD004203.

[5] Saint S, Greene MT, Krein SL, Rogers MAM, Ratz D, Fowler KE, et al. A program to prevent catheter-associated urinary tract infection in acute care. N Engl J Med 2016;374;2111-19.

[6] Alp E, Altun D, Cevahir F, Ersoy S, Cakir O, McLaws ML. Evaluation of the effectiveness of an infection control program in adult intensive care units: a report from a middle-income country. Am J Infect Control 2014;42;1056-61.

[7] Chenoweth CE, Saint S. Urinary tract infections. Infect Dis Clin North Am 2016;30;869-85.

[8] Jahn P, Beutner K, Langer G. Types of indwelling urinary catheters for long-term bladder drainage in adults. Cochrane Database Syst Rev 2012;10;CD004997. 
[9] Singha P, Locklin J, Handa H. A review of the recent advances in antimicrobial coatings for urinary catheters. Acta Biomater 2017;50;20-40.

[10] Al-Tawfiq JA, Amalraj A, Memish ZA. Reduction and surveillance of device-associated infections in adult intensive care units at a Saudi Arabian hospital, 2004-2011. Int J Infect Dis 2013;17;e1207-e11.

[11] Pfäfflin F, Tufa TB, Getachew M, Nigussie T, Schönfeld A, Häussinger D, et al. Implementation of the WHO multimodal hand hygiene improvement strategy in a University Hospital in Central Ethiopia. Antimicrob Resist Infect Control 2017;6;3.

[12] Chenoweth C, Saint S. Preventing catheter-associated urinary tract infections in the intensive care unit. Crit Care Clin 2013; 29;19-32.

[13] Farhoudi F, Sanaei Dashti A, Hoshangi Davani M, Ghalebi N, Sajadi G, Taghizadeh R. Impact of WHO hand hygiene improvement program implementation: a quasi-experimental trial. Biomed Res Int 2016;2016;7026169.

[14] Al Kuwaiti A. Impact of a multicomponent hand hygiene intervention strategy in reducing infection rates at a university hospital in Saudi Arabia. Interv Med Appl Sci 2017;9;137-43.

[15] Abdulmutalib DA, Abato AT, Mazi W, Senok A. P017: Reduction of catheter associated urinary tract infections following removal of unnecessary urinary catheters in a tertiary care hospital in Saudi Arabia. Antimicrob Resist Infect Control 2013;2;P17.

[16] Al-Hameed FM, Ahmed GR, AlSaedi AA, Bhutta MJ, Al-Hameed FF, AlShamrani MM. Applying preventive measures leading to significant reduction of catheter-associated urinary tract infections in adult intensive care unit. Saudi Med J 2018;39;97-102.

[17] Gaid E, Assiri A, McNabb S, Banjar W. Device-associated nosocomial infection in general hospitals, Kingdom of Saudi Arabia, 2013-2016. J Epidemiol Glob Health 2018;7;S35-S40.

[18] Rosenthal VD, Bat-Erdene I, Gupta D, Belkebir S, Rajhans P, Zand F, et al. International Nosocomial Infection Control Consortium (INICC) report, data summary of 45 countries for 2012-2017: device-associated module. Am J Infect Control 2020;48;423-32.

[19] Zaiton HI, Relloso JT, Medinah JM. Evaluating the impact of utilizing urinary catheter care bundle on minimizing the incidence of catheter-associated urinary tract infection (CAUTI) among intensive care patients. Am J Nurs Res 2019;7;836-45.

[20] Ghazal S, Viorica SC, Joseph M, Mukahal M, Hakawi A, Edmond $\mathrm{MB}$. Reducing the rate of catheter-associated urinary tract infection in the non-ICU setting. Adv Infect Dis 2015;5;81-6.

[21] Burton DC, Edwards JR, Srinivasan A, Fridkin SK, Gould CV. Trends in catheter-associated urinary tract infections in adult intensive care units-United States, 1990-2007. Infect Control Hosp Epidemiol 2011;32;748-56.

[22] Al Nasser W, El-Saed A, Al-Jardani A, Althaqafi A, Alansari H, Alsalman J, et al. Rates of catheter-associated urinary tract infection in tertiary care hospitals in 3 Arabian Gulf countries: a 6-year surveillance study. Am J Infect Control 2016;44;1589-94.

[23] Alharbi RD, Ali AHM, Almatroudi A, Mohamed S. The potential sources of transmitting of hospital acquired infection by routine devices in adult ICU in Alrass General Hospital. J Biosci Med 2020;8;69-80.

[24] Al-Tawfiq JA, Anani AA. Antimicrobial susceptibility pattern of bacterial pathogens causing urinary tract infections in a Saudi Arabian hospital. Chemotherapy 2009;55;127-31.

[25] Garcia R, Spitzer ED. Promoting appropriate urine culture management to improve health care outcomes and the accuracy of catheter-associated urinary tract infections. Am J Infect Control 2017;45;1143-53.

[26] Tenke P, Mezei T, Böde I, Köves B. Catheter-associated urinary tract infections. Eur Urol Suppl 2017;16;138-43.

[27] Sheik GB, Sannat A, Al-Rowis B, Jeddawy SM, Sherif DM. Prevalence, risk factors and antimicrobial susceptibility of beta lactamase producing enterobacteriacea in a tertiary care centre of Saudi Arabia. Int J Curr Microbiol Appl Sci 2016;5;98-109.

[28] Aljohi AA, Hassan HE, Gupta RK. The efficacy of noble metal alloy urinary catheters in reducing catheter-associated urinary tract infection. Urol Ann 2016;8;423-9.

[29] Al-Abdely HM, Alshehri AD, Rosenthal VD, Banjar W, Mohammed YK, Assiri AM, et al. Device-associated infection rates in Intensive Care Units of five cities of the Kingdom of Saudi Arabia: International Nosocomial Infection Control Consortium (INCC) findings. Can J Infect Control 2017;32;31-6. 